\title{
Carbon Nanotube Based Chemical Sensors for Space and Terrestrial Applications
}

\author{
Jing $\mathrm{Li}$ and Yijiang $\mathrm{Lu}$ \\ NASA Ames Research Center \\ Moffett Field, CA 94035
}

A nanosensor technology has been developed using nanostructures, such as single walled carbon nanotubes (SWNTs), on a pair of interdigitated electrodes (IDE) processed with a silicon-based microfabrication and micromachining technique. The IDE fingers were fabricated using photolithography and thin film metallization techniques. Both in-situ growth of nanostructure materials and casting of the nanostructure dispersions were used to make chemical sensing devices. These sensors have been exposed to nitrogen dioxide, acetone, benzene, nitrotoluene, chlorine, and ammonia in the concentration range of ppm to $\mathrm{ppb}$ at room temperature. The electronic molecular sensing of carbon nanotubes in our sensor platform can be understood by intra- and inter-tube electron modulation in terms of charge transfer mechanisms. As a result of the charge transfer, the conductance of p-type or hole-richer SWNTs in air will change. Due to the large surface area, low surface energy barrier and high thermal and mechanical stability, nanostructured chemical sensors potentially can offer higher sensitivity, lower power consumption and better robustness than the state-of-the-art systems, which make them more attractive for defense and space applications. Combined with MEMS technology, light weight and compact size sensors can be made in wafer scale with low cost. Additionally, a wireless capability of such a sensor chip can be used for networked mobile and fixed-site detection and warning systems for military bases, facilities and battlefield areas.

\section{Introduction}

The chemical sensor market has been projected to grow to $\$ 4$ billion worldwide within the next 10 years. Some of the primary motivations to develop nanostructured chemical sensors are monitoring and control of environmental pollution; improved diagnostics for point of care medical applications; reductions in measurement time, sensor size, and power consumption; improvement in measurement precision and accuracy; and improved detection limits for homeland security, battlefield environments, and process and quality control of industrial applications. In each of these applications, there is demand for sensitivity, selectivity and stability of environmental and biohazard detection and capture beyond what is currently commercially available. It is believed that the emerging field of nanotechnology can play an important role in realizing these goals ${ }^{1}$, and the prototype development described here represents a significant step in that direction. Sensors made of nanomaterials have promise for offering an enhanced generation of sensing devices that are smaller, consume less power, higher-performing, and less expensive that conventional sensors.

Nanotechnology offers the ability to work at the molecular level, atom by atom, to create large structures with fundamentally new molecular organization. It is essentially concerned with materials, devices, and systems whose structures and components exhibit novel and significantly improved physical, chemical and biological properties, phenomena, and process control due to their nanoscale size.

Carbon nanotubes, more than any other nanomaterials, have been generating much interest and excitement among researchers and the business and investment communities for use in variety of applications, such as extremely sensitive chemical and biochemical sensors, nano-scale computing devices, exceptionally strong fibers, hydrogen storage materials for fuel cells, batteries, optical devices, electronics, and catalysts. Carbon nanotubebased sensors have potential for detecting a single molecule of a substance and sensors using carbon nanotube material could be extremely small (on the order of 1,000 times smaller than the size of a MEMS sensor), and possibly offer orders of magnitude less power consumption than a MEMS sensor.

Moreover, carbon nanotube technology is particularly suitable and promising for chemical detection, since the technology can be applied to offer gas or liquid chemical sensors that would have extremely low power, ultraminiature size, versatility, as well as cost benefits. Such low power carbon nanotube sensors would facilitate distributed, or wireless, gas sensing, leading to more efficient multi-point measurements, or greater convenience and flexibility in performing measurements. In addition, carbon nanotube chemical sensors are suitable for sensing different analytes of interest to the user; and such sensors could be configured in the form of an array to comprehensively and cost-effectively monitor multiple analytes.

One such nanotechnology-enabled chemical sensor has been developed at NASA Ames leveraging nanostructures, such as single walled carbon nanotubes (SWNTs) and metal oxide nanobelts or nanowires, as a sensing medium bridging a pair of interdigitated electrodes (IDE) realized through a silicon-based microfabrication 
and micromachining technique. The IDE fingers are fabricated on a silicon substrate using standard photolithography and thin film metallization techniques. It is noteworthy that the fabrication techniques employed are not confined to the silicon substrate. Through spin casting and careful substrate selection (i.e. clothing, glass, polymer, etc.), additional degrees of freedom can be exploited to enhance sensitivity or to conform to unique applications.

Sensors developed have been exposed to nitrogen dioxide, acetone, benzene, nitrotoluene, chlorine, and ammonia and repeatedly sensed these analytes in the ppm to $\mathrm{ppb}$ concentration range at room temperature. Due to their large surface area, low surface energy barrier and high thermal and mechanical stability, nanostructured chemical sensors offer higher sensitivity, lower power consumption and a more robust solution than most state-ofthe-art systems making them attractive for defense and space applications, as well as a variety of commercial applications. Leveraging micromachining technology, light weight and compact sensors can be fabricated, in wafer scale, with high yield and at low cost. Additionally, the wireless potential of such sensors can be leveraged to network mobile and fixed-base detection and warning systems for civilian population centers, military bases and battlefields, as well as other high-value or high-risk assets and areas in industry.

\section{Experimental}

A. Single CNT device and multi-CNTs device

Single carbon nanotube field effect transistor was fabricated for being an electronic component by a group at $\mathrm{IBM}^{2}$, and Dekker at Delft ${ }^{3}$. Soh ${ }^{4}$ and $\mathrm{Dai}^{5}$ have combined the synthesis of carbon nanotube and microfabrication of the electrodes on a silicon ( $\mathrm{Si}$ ) substrate that is used as a back gate. There are four steps, with electron beam lithography using polymethylmethacrylate as the resist, involved in this device fabrication process: 1) patterning of the electrodes windows ( $5 \mu \mathrm{m} \times 5 \mu \mathrm{m})$ using alignment marks on the Si substrate; 2 ) forming the catalyst islands in the electrode windows for carbon nanotube growth; 3) growing the carbon nanotubes via methane CVD to bridge the islands; 4) Patterning and depositing the Ti/Au over the catalyst islands for electrical contact pads. The operation of these FETs was found to depend heavily on the ambient conditions, thus leaving to the evolution of the nanotube FET for sensing applications. Since then, these have been demonstrations of chemical sensing of $\mathrm{NO}_{2}$, ammonia etc. ${ }^{6}$. While a single SWNT retains the advantages of sensitivity expected from a nanotube, fabrication of a CNT FET is relatively complex; it is particularly tedious to grow one semiconducting SWNT by CVD to bridge the contacts and yield is poor.

\section{B. Sensing devices with carbon nanotube mesh or thin film}

Another way of making a sensing device with carbon nanotubes is to grow the nanotubes somewhere else first, and then place them onto a device. $\mathrm{Li}$ and colleagues have developed a gas sensor based on an interdigitated electrode (IDE) design with carbon nanotubes forming a network type of thin layer laid across the electrodes (see Fig. 1a). The conductivity of the CNT network changes upon exposure of the ambient that containing the gas or vapor $^{7}$. The device fabrication process involved two simple steps: 1) making interdigitated electrodes (10 $\mu \mathrm{m}$ of finger width and $8 \mu \mathrm{m}$ of gap between fingers) of Ti/Au on silicon substrate using conventional lithography; 2) dispersing the purified carbon nanotubes ${ }^{8}$ in a solvent and dispense a droplet onto the IDE area (see figure 1a).

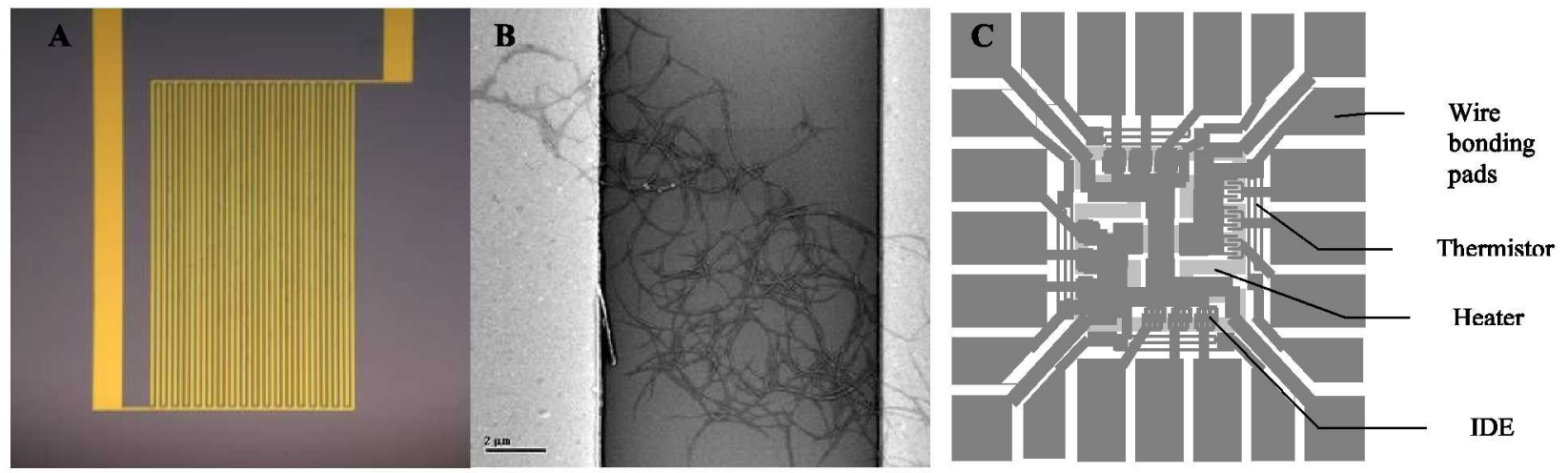

Figure 1. SWNTs across the fingers of an interdigitated electrode: A) Photo image of an IDE; B) SEM image of SWNTs across two gold electrodes, C) An array of 12 IDEs on a $1 \times 1 \mathrm{~cm}^{2}$ silicon chip with heater and thermistor.

The sensor performance can be tuned by varying the IDE finger gap size and the density of the carbon nanotube across the electrodes by varying the concentration of the carbon nanotube dispersion. This is a simple 
process with a high sensor yield, which can be scaled up for mass production. This technology developed at NASA Ames Research Center has attracted much of attention from industry and Integrated Nanosystems, Inc. is commercializing this technology for a variety of applications.

There are several other deposition methods useful in sensor fabrication such as spin-coating, ink jetting, dipping-coating, and air brushing. These methods can easily provide carbon nanotube sensors with different chemical functionalities forming a sensor array. Carbon nanotubes can be pretreated by several methods: 1) modifying the surface of the nanotubes with functional groups, such as carboxylic group for base molecules, ammine groups for acidic molecules and other aromatic groups for large organic molecules; 2) Doping the carbon nanotubes with catalytic metal clusters such as $\mathrm{Pd}, \mathrm{Pt}$ and $\mathrm{Au}$ for hydrogen and hydrocarbon, and $\mathrm{Cu}$ and $\mathrm{Rh}$ for nitric compounds. In this case, the carbon nanotube ensemble will act as a matrix that holds the metal binding sites for chemical sensing; 3 ) coating the surface of carbon nanotubes with different polymers - such as polystyrene, polyvinylalcohol, etc. used in commercial polymer based chemical sensing array for organic vapor detection - that provides specific interactions with chemical species of interest.

The same approach of putting down the carbon nanotubes has been used by Collins ${ }^{9}$ to form a gas sensor using the thin film of single walled carbon nanotube (SWNTs) deposited by its dispersion in dichloroethane; by Sumanasekera ${ }^{10}$ to fabricate gas sensors for detection of hydrocarbons; by Chopra ${ }^{11}$ and $\mathrm{Ong}^{12}$ for making resonantcircuit sensors using multiwalled carbon nanotubes (MWNTs) for ammonia and carbon dioxide detection.

The fabricated sensor array chips were tested using a custom made multi-channel data acquisition system for a sensor array that has more 32 sensing elements, and a data acquisition card (DaqBoad/2000, IOtech, Inc. Cleveland, Ohio) and an adapter board (DBK203, IOtech, Inc. Cleveland, Ohio) with screw terminals, also connects DBK signal conditioning and expansion options to a CA-195 expansion cable. A readout chip, separate from the sensor array chip, contained a Wheatstone bridge readout circuitry was built for boost and massage the electrical signals from the sensor through the data acquisition card. This circuitry measured the voltage shift from the original excitation voltage when the gas exposed to the sensor. Three channels were multiplexed during the measurement. The fabricated sensor array chips were exposed to gases at various concentrations using a computerized multicomponent gas blending and dilution system, Environics 2040 (Environics, Inc. Tolland, CT). It created different concentration streams with a steady output flow of $400 \mathrm{cc} / \mathrm{min}$ during both exposure and purge periods. Air was used as the purge and the balance gas.

\section{Results and Discussions}

Our experimental results with carbon nanotubes indicate a conductivity change with exposure to $\mathrm{NO}_{2}$ at different concentrations and the detection limit of it is $44 \mathrm{ppb}$ (see figure 1), and to nitrotoulene (simulate of explosives) with a detection limit of $254 \mathrm{ppb}$, as well as a variety of other organic and inorganic vapors and gases (see figure 2). Preliminary results also show sensitivity in the ppb range with reproducible sensitivity within $6 \%$ between sensors. By loading the carbon nanotubes with catalytic metal nano clusters and coating polymers on the surface of carbon nanotubes, excellent selectivity was achieved for room temperature detection of nitrogen dioxide in sub ppb, methane (combustible) in sub ppm and chlorine (toxic) gas in ppb level.

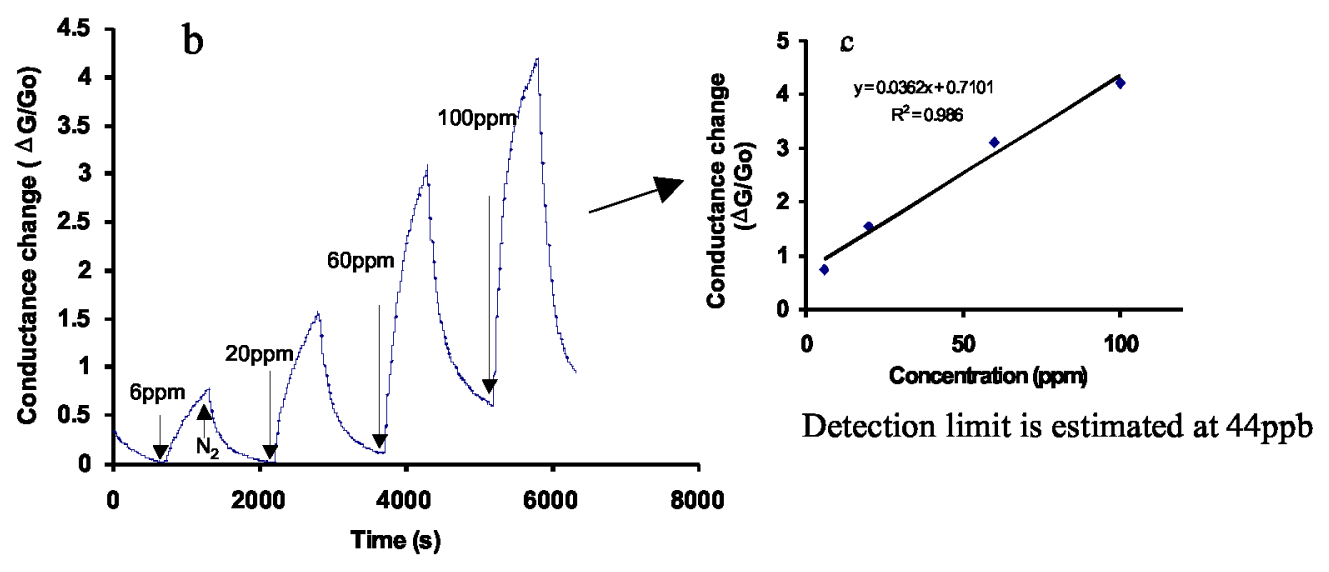

Figure 1. Representative sensor response for $\mathrm{NO}_{2}$ in a $400 \mathrm{cc} / \mathrm{min}$. total flow at room temperature 


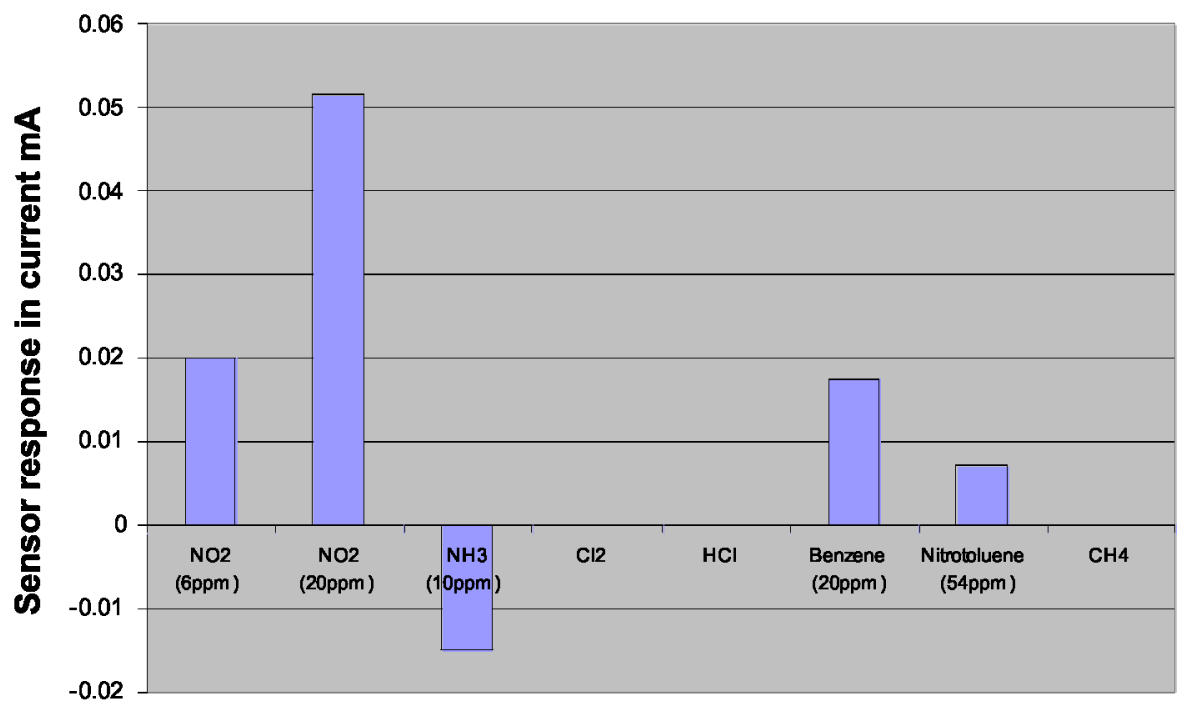

Different gases with concentration in ppm

Figure 2a: Comparison of carbon nanotube sensors to different gases and vapors

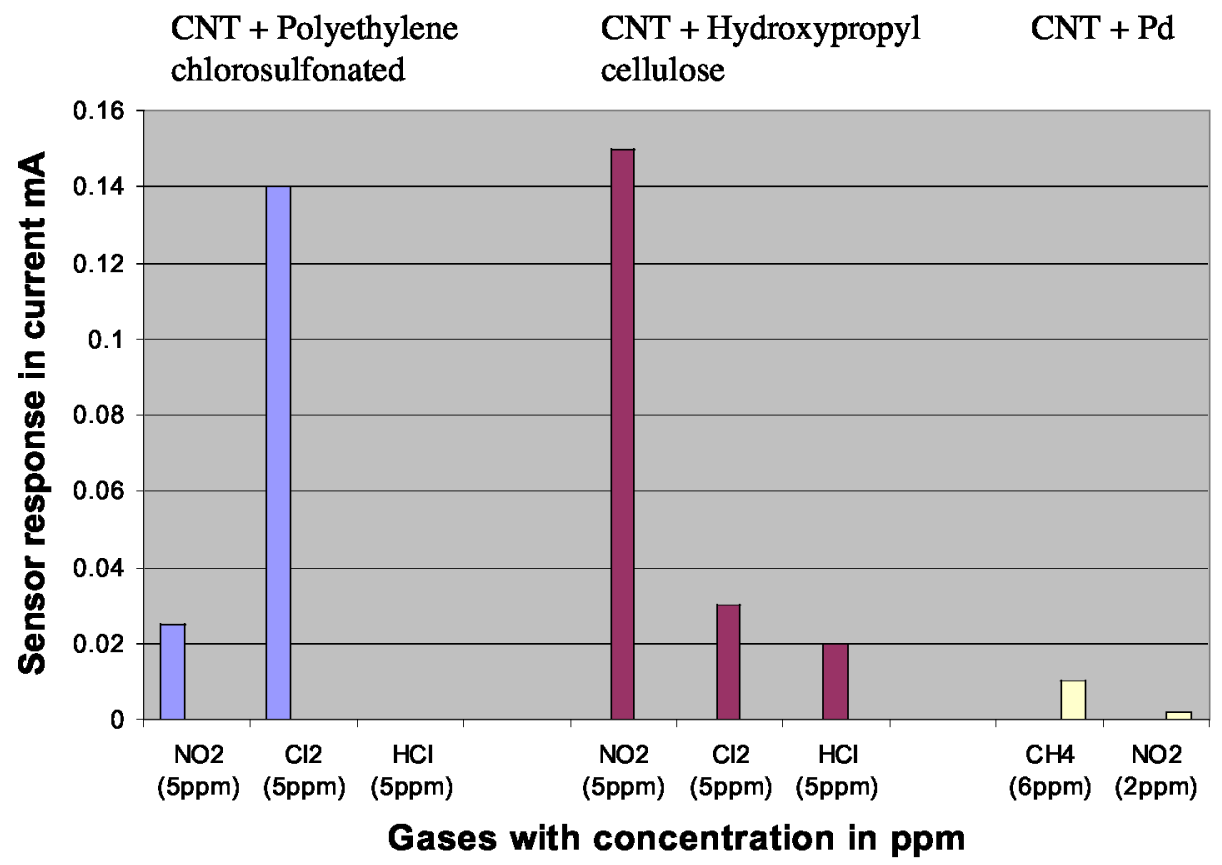

Figure 2b: Comparison of CARBON NANOTUBES with different mixing materials for gases

Combining the carbon nanotube-based chemical sensor with MEMS technology, a lab-on-a-chip can be built, which has onboard sampling system, data processing and potentially wireless communication capabilities. Such a lab-on-a-chip system can be deployed across a wide spectrum of hardware platforms for environmental monitoring, 
and can be made into a portable handheld device (see figure 3). The handheld device will have a built-in LCD to display the identified chemical for easy of use by end user.

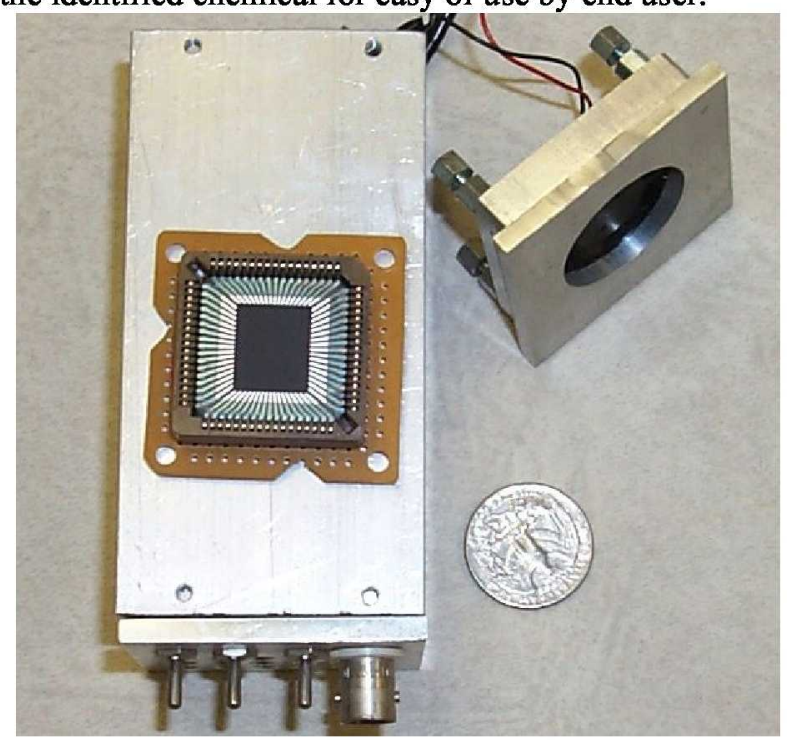

Figure 3: A handheld device with a nanostructure-based chemical sensor array for in-situ chemical detection

Based on this nanosensor development described above, a 32-channel sensor array module loaded with different nanostructures has been developed for a space flight demonstration (see figure 4). This module has been launched via a Navy satellite in the Earth orbit at around $500 \mathrm{kM}$ and repeatedly demonstrated the trace $\mathrm{NO}_{2}$ detection.

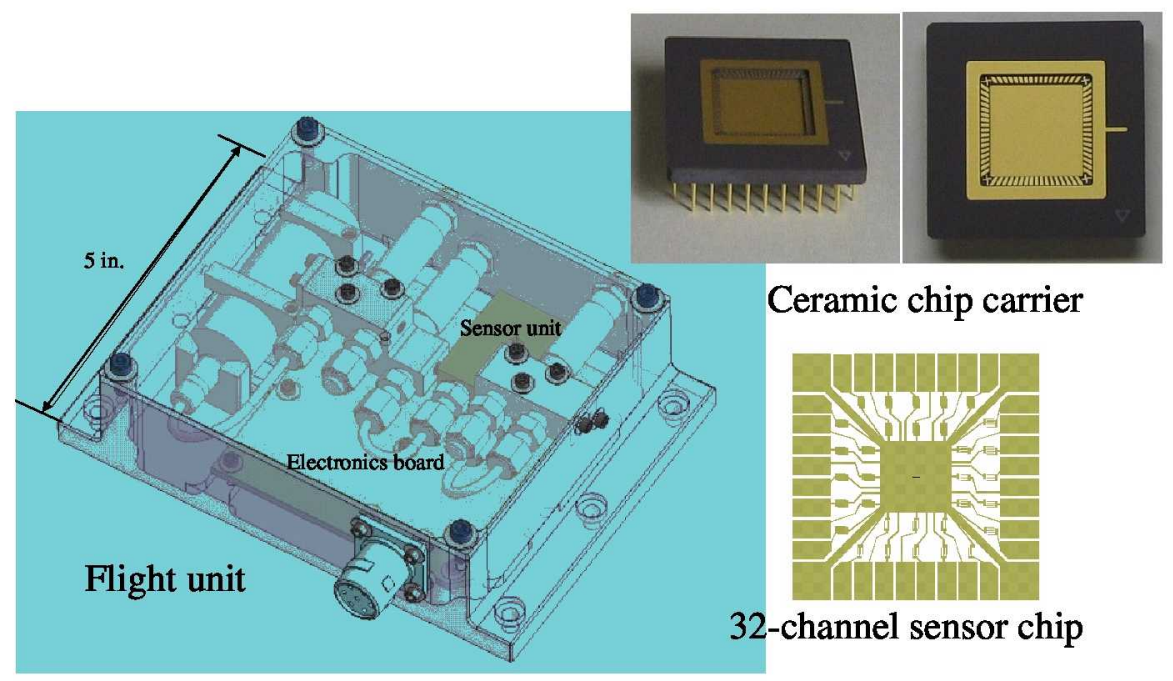

Figure 4: A nanostructure-based multi-sensor module for a flight demonstration in space

Compared with other nanotechnology based chemical sensors discussed above, our design of the nanostructurebased chemical sensor uses an interdigitated electrode platform, and variety nanostructured materials for chemical sensing with following advantages:

- High sensitivity (current detection proved to be $44 \mathrm{ppb}$ for $\mathrm{NO}_{2}$, and potentially singlemolecule/quantum-capture sensitivity) due to their well organized molecular structure and extraordinarily high surface to volume ratio,

- Wide range of detectable chemical analytes,

- Tunable sensing properties through manipulation of nanostructured materials for selectivity, 
- Small size and lightweight.

- Reliable sensor performance from chip to chip,

- High yield and scalable sensors manufacturability, and low cost for mass production,

- Lower power consumption ( $\mu \mathrm{W}$ to $\mathrm{mW}$ ), which is ideal for wireless monitoring, persistent surveillance and environmental monitoring applications,

- Simple sensor design uses microfabrication, amenable for cost-effective scale up,

- Simple electronic design for easy measurement and integration,

- Capability of built-in intelligence onto the sensor chip, such as on board data processing, and sample inlet and outlet system,

- Having the variety of sensing materials, this universal IDE platform sensor array can be utilized for different application and extend to the different end products, such as network-distributed sensors, handheld devices, or sensor module for plug and play.

\section{Applications}

Sensor development using CNTs is at its early stages. The potential in various sectors is outlined below. The challenges are common to all these sectors: inexpensive fabrication steps, sensor robustness, reliability, reproducibility, and system integration.

Industry

Carbon nanotube based chemical sensors possess high sensitivity, small size and low power consumption, which can be used to quickly verify incoming raw materials at the delivery point. The technology can significantly reduce the amount of time and money spent analyzing those materials in a lab, as well as reducing the amount of materials handling. Most changes in chemical processes can be reflected in the changing composition of the vapor phase surrounding or contained within the process. Thus, the vapor phase sensors enable the quick assessment of the chemical status of most industrial processes. Examples are found across many sectors including food processing (coffee roasting and fermentation), petrochemical (plastics manufacture and gasoline blending) and consumer products (detergents and deodorants). Much like vision inspection is used to assess the visual integrity (color, shape, size) of products, olfactory inspection assesses the chemical integrity (consistency, presence of contaminants).

\section{Environment}

Increasing awareness and new regulations for safety and emission control make environmental monitoring one of the most desired amongst the numerous industrial and civil applications for which the development of reliable solid-state gas sensors is demanded. Current methods for air quality control approved by the standards consist of analytical techniques, which need the use of very costly and bulky equipment. For applications in this arena, sensors that are able to selectively detect various gases at a concentration level of a few ppb and in the form of low-cost portable handheld devices for continuous in-situ monitoring are needed. With unique advantages of high sensitivity, small size and low power consumption, and strong mechanical and thermal stability, carbon nanotube based chemical sensors are best fit for this type of application.

Defense

Chemical sensors are very focused for security and defense applications due to their portability and low power consumption. Carbon nanotube sensors potentially can offer higher sensitivity and lower power consumption than the state-of-the-art systems, which make them more attractive for defense applications. Some examples include monitoring filter breakthrough, personnel badge detectors, embedded suit hermiticity sensors and other applications. Additionally, a wireless capability with the sensor chip can be used for networked mobile and fixed-site detection and warning systems for military bases, facilities and battlefield areas.

Medical/Bio

It is believed that chemical sensors would provide physicians with a quicker and more accurate diagnostic tool. Applications could include obtaining objective information on the identity of certain chemical compounds in exhaled air and excreted urine or body fluids related to specific metabolic conditions, certain skin diseases or bacterial infections, such as those common to leg or burn wounds. Additionally, the chemical sensors may provide more accurate, real-time patient monitoring during anesthesia administration.

\section{Acknowledgments}

This work is funded by NASA Bio-Nano program. Author also would like to thank Dr. Harry Partridge, Meyya Meyyappan, Jim Arnold and Dan Powell for their management support. 


\section{References}

${ }^{1}$ Li, J., Chemical and Physical Sensors, in Carbon Nanotubes: Science and Applications, Editor: M. Meyyappan, CRC Press, Boca Raton, FL, USA, 2004.

${ }^{2}$ Collins, P. G., Amold, M.S., Avouris, P., Science, Vol. 292, 2001, 706.

${ }^{3}$ Tans, S.J., Verschueren, A. R. M., Dekker, C., Nature, Vol. 393, 1998, 49.

${ }^{4}$ Soh, H. T., Quate, C. F., Morpurgo, A. F., Marcus, C. M., Kong, J., and Dai, H., Appl. Phys. Lett., Vol. 75, $1999,627$.

${ }^{5}$ Dai, H., Kong, J., Zhou, C., Franklin, N., Tombler, T., Cassell, A., Fan, S., and Chapline, M., J. Phys. Chem. B, Vol. 103, 1999, 11246.

${ }^{6}$ Kong, J., Franklin, N. R., Zhou, C., Chapline, M. G., Peng, S., Cho, K., Dai, H., Science, Vol. 287, $2000,622$.

${ }^{7} \mathrm{Li}$, J., Lu, Y., Ye, Q., Cinke, M., Han, J., and Meyyappan, M., Nano Lett., Vol. 3, 2003, 929.

${ }^{8}$ Cinke, M., Li, J., Chen, B., Cassell, A., Delzeit, L, Han, J., and Meyyappan, M., Chem. Phys. Lett. Vol. 365, $2002,69$.

${ }^{9}$ Collins, P.G., Bradley, K., Ishigami, M., and Zettl, A., Science, Vol. 287, 2000, 1801.

${ }^{10}$ Sumanasekera, G.U., Adu, C.K.W., Fang, S., Eklund, P.C., Phys. Rev. Lett. Vol. 85, $2000,1096$.

${ }^{11}$ Chopra, S., Pham, A., Gaillard, J., Parker, A., Rao, A. M., Appl. Phys. Lett. Vol. 80, 2002, 4632.

${ }^{12}$ Ong, K. G., Zeng, K., Grimes, G.A., IEEE sens. J. Vol. 2, 2002, 82. 